\title{
Dynamical analysis on a single population model with state-dependent impulsively unilateral diffusion between two patches
}

\author{
Jianjun Jiao ${ }^{1 *}$, Lansun Chen ${ }^{2}$ and Shaohong Cai ${ }^{1}$
}

"Correspondence:

jiaojianjun05@126.com

${ }^{1}$ School of Mathematics and

Statistics, Guizhou College of

Finance and Economics, Guiyang,

550004, P.R. China

Full list of author information is

available at the end of the article

\begin{abstract}
Coupling with social progress, traffic development and so on, the natural environment is going into patches. There are differences between population in differential patches. The population living in a patchy environment is affected by the space structures. It is important to depict the dynamical behaviors of the population in the present world. In this work, a state-dependent impulsive differential model, which focuses on impulsively unilateral diffusion between two patches, aims for the simulation of the factual population dynamical behaviors. With the approaches of mathematical analysis, we obtain sufficient conditions of the existence and orbitally asymptotic stability of a periodic solution of the investigated system. Finally, the numerical simulations verify our results.
\end{abstract}

Keywords: single population; state-dependent impulses; unilateral diffusion; patchy environment

\section{Introduction}

The population diffusion affecting the dynamical behaviors of populations in differential patches are investigated by many researchers [1-5]. Two unable to be competitive populations can be stabilized by the population diffusion [1]. The persistence of competitive systems of two or three populations can be increased under appropriate diffusion conditions [5]. Some papers assume that the individuals' mobility is mainly induced by intrinsic factors of population such as genetic characteristics. Nevertheless, more and more researchers have found that the population diffusion could be affected by many factors such as qualities and quantities of food, the pressures from competitors, and the predation risk from enemies. Abrams et al. [6] found that prey diffusion may be accelerated by poor reproduction conditions and high predation risks in local habitat. Because of attraction from better reproductions or less predation pressure at other patches, the predator may change behaviors on the basis of prey abundance and demographic advantages. Kuang [7] analyzed a delayed two-stage population model with space limited recruitment.

The theory of impulsive differential equations is much richer than the corresponding theory of differential equations. They generally describe phenomena which are subjected to steep or instantaneous changes. Impulsive equations are found in almost every domain of the applied science and have been studied in many works [8, 9]. Jiao et al. [10] and Tang et al. [11] have studied the population models with impulsive perturbation at fixed mo-

(c) 2012 Jiao et al.; licensee Springer. This is an Open Access article distributed under the terms of the Creative Commons Attribution License (http://creativecommons.org/licenses/by/2.0), which permits unrestricted use, distribution, and reproduction in any medium, provided the original work is properly cited. 
ments. Jiao et al. [12] investigated the dynamical behaviors of a stage-structured predatorprey system with birth pulse and impulsive harvesting at different moments. Jiao et al. [13] provided a fishing policy by investigating a stage-structured model with state-dependent impulsive harvesting.

Coupling with social progress, traffic development and so on, the natural environment is going into patches. There are differences between population in differential patches. For example, when the density of population living in one patch reaches one threshold, that is to say, when the quantity of population reaches the superior limit, intraspecific competition for limited resources increases, the population in this patch will move to another patch. Although Jiao et al. [14] devoted their work to investigation of the dynamics of a stage-structured predator-prey model with prey impulsively diffusing between two patches, they did not propose a single population model with statedependent impulsively unilateral diffusion between two patches. Motivated by these biological facts and the previous studies, we propose and investigate a state-dependent impulsive differential model, which focuses on impulsively unilateral diffusion between two patches.

The organization of this paper is as follows. In the next section, we introduce the model and background concepts. In Section 3, some important lemmas are presented. In Section 4 , we give the sufficient conditions of the existence and orbitally asymptotic stability of a periodic solution of the investigated system. In Section 5, numerical simulation and a brief discussion are given to conclude this work.

\section{The model}

We propose a state-dependent impulsive differential model which focuses on impulsively unilateral diffusion between two patches

$$
\left\{\begin{array}{l}
\frac{d x_{1}(t)}{d t}=x_{1}(t)\left(a-b x_{1}(t)\right), \\
\frac{d x_{2}(t)}{d t}=-c x_{2}(t), \\
\triangle x_{1}(t)=-d x_{1}(t), \\
\triangle x_{2}(t)=d x_{1}(t),
\end{array}\right\} x_{1}(t)<x_{1}^{*},
$$

where system (2.1) is assumed to be composed of two patches. $x_{i}(t)(i=1,2)$ denotes the density of population in $i$ th patch. $a>0$ denotes the intrinsic rate of natural increase in the population in Patch 1. $b>0$ denotes the interspecific competition coefficient of the population in Patch 1. $c>0$ denotes the death rate of the population in Patch $2.0<d<1$ denotes the diffusive rate coefficient from Patch 1 to Patch $2 . x_{1}^{*}>0$ is called an environment pressure threshold of the population in Patch 1, that is to say, when the density of the population in Patch 1 reaches the threshold $x_{1}^{*}$, the population will unilaterally diffuse from Patch 1 to Patch 2. It is also assumed that the population in Patch 2 does not diffuse from Patch 2 to Patch 1. 


\section{The definitions and lemmas}

Before starting our work, we need two definitions and two lemmas. Consider the autonomous impulsive differential equation

$$
\begin{cases}\frac{d x}{d t}=f(x, \varepsilon), & x \notin \sigma(\varepsilon), \\ \triangle x=I(x, \varepsilon), & x \in \sigma(\varepsilon),\end{cases}
$$

where $\varepsilon \in J=(-\bar{\varepsilon}, \bar{\varepsilon})$ is a small parameter. For each $\varepsilon \in J$, the $\sigma(\varepsilon)$ is a hypersurface in $R^{n}$. Suppose $\sigma(\varepsilon)$ consists of $q$ nonintersecting smooth hypersurfaces $\sigma_{k}(\varepsilon)$ which are given by the equations $\varphi_{k}(x, \varepsilon)=0(k=1,2, \ldots, q)$.

Let $x=\phi(t), t \in R_{+}$be a solution of Equation (3.1) with moments of impulsive effect $\tau_{k}: 0<\tau_{1}<\tau_{2}<\cdots, \lim _{k \rightarrow \infty} \tau_{k}=+\infty$, and $L_{+}=\left\{x \in R^{n}: x=\phi(t), t \in R_{+}\right\}$. Let $x\left(t, t_{0}, x_{0}\right)$ denote the solution of Equation (3.1) for which $x\left(t^{+}, t_{0}, x_{0}\right)=x_{0}$, and let $J^{+}\left(t_{0}, x_{0}\right)$ denote the right maximal interval of the existence of this solution.

Lemma 3.1 The solution $x=\phi(t)$ of Equation (3.1) is said to be

(i) orbitally stable, if

$$
\begin{aligned}
& (\forall \rho>0)(\forall \eta>0)\left(\forall t_{0} \in R_{+},\left|t-\tau_{k}\right|>\eta\right)(\exists \delta>0) \\
& \left(\forall x_{0} \in R^{n}, d\left(x_{0}, L_{+}\right)<\delta, x_{0} \notin B_{\eta}\left(\phi\left(\tau^{+}\right)\right) \cup B_{\eta}(\phi(\tau))\right)\left(\forall t \in J^{+}\left(t_{0}, x_{0}\right)\right) \\
& d\left(x\left(t, t_{0}, x_{0}\right), L_{+}\right)<\rho ;
\end{aligned}
$$

(ii) orbitally attractive, if

$$
\begin{aligned}
& (\forall \eta>0)\left(\forall t_{0} \in R_{+},\left|t-\tau_{k}\right|>\eta\right)(\exists \lambda>0) \\
& \left(\forall x_{0} \in R^{n}, d\left(x_{0}, L_{+}\right)<\lambda, x_{0} \notin B_{\eta}\left(\phi\left(\tau^{+}\right)\right) \cup B_{\eta}(\phi(\tau))\right)\left(\forall t \in J^{+}\left(t_{0}, x_{0}\right)\right)(\forall \rho>0) \\
& \left(\exists \sigma>0, t_{0}+\sigma \in J^{+}\left(t_{0}, x_{0}\right)\right)\left(\forall t \geq t_{0}+\sigma, t \in J^{+}\left(t_{0}, x_{0}\right)\right) \\
& d\left(x\left(t, t_{0}, x_{0}\right), L_{+}\right)<\rho ;
\end{aligned}
$$

(iii) orbitally asymptotically stable, if it is orbitally stable and orbitally attractive.

Definition 3.2 The solution $x=\phi(t)$ of Equation (3.1) is said to enjoy the property of asymptotic phase if

$$
\begin{aligned}
& (\forall \eta>0)\left(\forall t_{0} \in R_{+},\left|t-\tau_{k}\right|>\eta\right)(\exists \lambda>0) \\
& \left(\forall x_{0} \in R^{n},\left|x_{0}-\phi\left(t_{0}\right)\right|<\lambda\right)(\exists c \in R)(\forall \rho>0) \\
& \left(\exists \sigma>|c|, t_{0}+\sigma \in J^{+}\left(t_{0}, x_{0}\right)\right)\left(\forall t>t_{0}+\sigma, t \in J^{+}\left(t_{0}, x_{0}\right),\left|t_{0}-\tau_{k}\right|>\eta\right) \\
& \left|x\left(t, t_{0}, x_{0}\right)-\phi(t)\right|<\rho .
\end{aligned}
$$

Lemma 3.3 [15] For Equation (3.1) with $\varepsilon>0$, the following conditions hold:

$\left(c_{1}\right)$ For $\varepsilon=0$, Equation (3.1) has a $\tau_{0}$-periodic solution $x=\phi(t)$ with moments of impulsive effect $\tau_{k}: \tau_{k+q}=\tau_{k}+\tau_{0}(k \in Z)$ and $\phi^{\prime}(t) \not \equiv 0(t \in R)$. 
$\left(c_{2}\right)$ For each $k=1,2, \ldots, q$, the function $\varphi(x, \varepsilon)$ is differentiable in some neighborhood of the point $\left(\phi\left(\tau_{k}\right), 0\right)$ and

$$
\varphi_{k}\left(\phi\left(\tau_{k}\right), 0\right)=0, \quad \frac{\partial \varphi_{k}}{\partial x}\left(\phi\left(\tau_{k}\right), 0\right) f\left(\phi\left(\tau_{k}\right), 0\right) \neq 0
$$

(c $\left.c_{3}\right)$ There exists a $\delta>0$ such that for each $\varepsilon \in(-\delta, \delta)$ and $x_{0} \in R^{n},\left|x_{0}-\phi(0)\right|<\delta$, the solution $x\left(t, x_{0}, \varepsilon\right)$ of Equation (3.1) is defined for $t \in\left[0, \tau_{0}+\delta\right]$. Let the multipliers $\mu_{j}(j=$ $1,2, \ldots, n)$ of the variational equation

$$
\left\{\begin{array}{l}
\frac{d z}{d t}=\frac{\partial f(\phi(t), 0)}{\partial x} z, \quad t \neq \tau_{k} \\
\triangle z=N_{k} z, \quad t=\tau_{k}
\end{array}\right.
$$

where

$$
\begin{aligned}
N_{k}= & \frac{\partial I}{\partial x}\left(\phi\left(\tau_{k}\right), 0\right) \\
& +\left[f\left(\phi\left(\tau_{k}^{+}\right), 0\right)-f\left(\phi\left(\tau_{k}\right), 0\right)\left(1+\frac{\partial I}{\partial x}\left(\phi\left(\tau_{k}\right), 0\right)\right)\right] \frac{\frac{\partial \varphi}{\partial x}}{\frac{\partial \varphi}{\partial x} f},
\end{aligned}
$$

satisfy the condition

$$
\mu_{1}=1, \quad\left|\mu_{j}\right|<1 \quad(j=2,3, \ldots, n),
$$

then the $\tau_{0}$-periodic solution $x=\phi(t)$ of Equation (3.1) with $\varepsilon=0$ is orbitally asymptotically stable and enjoys the property of asymptotic phase.

If $n=2$, Equation (3.1) has the form

$$
\left\{\begin{array}{l}
\frac{d x}{d t}=P(x, y), \\
\frac{d y}{d t}=Q(x, y), \\
\triangle x=\alpha(x, y), \\
\triangle y=\beta(x, y),
\end{array}\right\} \varphi(x, y)=0 .
$$

If Equation (3.3) has a $\tau_{0}$-periodic solution $x=\zeta(t), y=\eta(t)$ and the condition of Lemma 3.1 are satisfied, then it can be (check [13]) that the corresponding variational system has multipliers $\mu_{1}$ and

$$
\mu_{2}=\prod_{k=1}^{q} \triangle_{k-1} \exp \left\{\int_{0}^{\tau_{0}}\left(\frac{\partial P}{\partial x}(\zeta(t), \eta(t))+\frac{\partial Q}{\partial y}(\zeta(t), \eta(t))\right) d t\right\}
$$

where

$$
\triangle_{k}=\frac{P_{+}\left(\frac{\partial \beta}{\partial y} \frac{\partial \phi}{\partial x}-\frac{\partial \beta}{\partial x} \frac{\partial \phi}{\partial y}+\frac{\partial \phi}{\partial x}\right)+Q_{+}\left(\frac{\partial \alpha}{\partial x} \frac{\partial \phi}{\partial y}-\frac{\partial \alpha}{\partial y} \frac{\partial \phi}{\partial x}+\frac{\partial \phi}{\partial y}\right)}{P \frac{\partial \phi}{\partial y}+Q \frac{\partial \phi}{\partial y}},
$$

and $P, Q, \frac{\partial \alpha}{\partial x}, \frac{\partial \alpha}{\partial y}, \frac{\partial \beta}{\partial x}, \frac{\partial \beta}{\partial y}, \frac{\partial \phi}{\partial x}, \frac{\partial \phi}{\partial y}$ are calculated at point $\left(\zeta\left(\tau_{k}\right), \eta\left(\tau_{k}\right)\right)$ and $P_{+}=P\left(\zeta\left(\tau_{k}^{+}\right)\right.$, $\left.\eta\left(\tau_{k}^{+}\right)\right), Q_{+}=Q\left(\zeta\left(\tau_{k}^{+}\right), \eta\left(\tau_{k}^{+}\right)\right)$. 
Lemma 3.4 [13] The T-periodic solution $(x(t), y(t))=(\zeta(t), \eta(t))$ of system (3.3) is orbitally asymptotically stable and enjoys the property of asymptotic phase if the multiplier $\mu_{2}$ calculated by (3.4) satisfies $\left|\mu_{2}\right|<1$.

\section{The dynamical analysis}

Set

$$
\tau=\min \left\{t>0: x_{1}(t)=x_{1}^{*}\right\} .
$$

Theorem 4.1 If

$$
(1-d) e^{a \tau}>1
$$

system (2.1) has a uniquely $\tau$-periodic solution.

Proof In view of the impulsive effect of system (2.1), if $x_{1}(0)=x_{1}\left(\tau^{+}\right)$and $x_{2}(0)=x_{2}\left(\tau^{+}\right)$, there exists a $\tau$-periodic solution of system (2.1). It is easy to calculate

$$
\left\{\begin{array}{l}
x_{1}(t)=\frac{a x_{1}(0) e^{a t}}{a+b x_{1}(0)\left(e^{a t}-1\right)}, \\
x_{2}(t)=x_{2}(0) e^{-c t},
\end{array}\right.
$$

with the initial conditions $\left(x_{1}(0), x_{2}(0)\right)$. When $t=\tau$, we obtain

$$
\left\{\begin{array}{l}
x_{1}(\tau)=\frac{a x_{1}(0) e^{a \tau}}{a+b x_{1}(0)\left(e^{a \tau}-1\right)} \\
x_{2}(\tau)=x_{2}(0) e^{-c \tau}
\end{array}\right.
$$

In view of the effect of the state-dependent impulsively unilateral diffusion of system (2.1) as

$$
\Delta x_{1}(t)= \begin{cases}0, & x_{1}(t)<x_{1}^{*}, \\ -d x_{1}(t), & x_{1}(t)=x_{1}^{*}\end{cases}
$$

and

$$
\Delta x_{2}(t)= \begin{cases}0, & x_{1}(t)<x_{1}^{*}, \\ d x_{1}(t), & x_{1}(t)=x_{1}^{*},\end{cases}
$$

we have

$$
\left\{\begin{array}{l}
x_{1}\left(\tau^{+}\right)=(1-d) x_{1}(\tau), \\
x_{2}\left(\tau^{+}\right)=x_{2}(\tau)+d x_{1}(\tau) .
\end{array}\right.
$$

Considering $x_{1}(0)=x_{1}\left(\tau^{+}\right)$and $x_{2}(0)=x_{2}\left(\tau^{+}\right)$, and substituting (4.3) into (4.4) and (4.6), we get $\left(x_{1}(0), x_{2}(0)\right)=\left(\frac{a\left[(1-d) e^{a \tau}-1\right]}{b\left(e^{a \tau}-1\right)}, \frac{d a\left[(1-d) e^{a \tau}-1\right]}{(1-d) b\left(e^{a \tau}-1\right)\left(1-e^{-c \tau}\right)}\right)$, and $\left(x_{1}(0), x_{2}(0)\right)=(0,0)$. From the impulsive effect $(1-d) x_{1}^{*}=x_{1}(0)$, we cast out $\left(x_{1}(0), x_{2}(0)\right)=(0,0)$. This completes the proof. 
Remark 4.2 From Theorem 4.1 and $(1-d) x_{1}^{*}=x_{1}(0)$, we can easily calculate

$$
\tau=\frac{1}{a} \ln \frac{(1-d) b x_{1}^{*}-a}{(1-d)\left(b x_{1}^{*}-a\right)} .
$$

Theorem 4.3 If condition (4.1) and

$$
\frac{(1-d)\left[a-b(1-d) x_{1}^{*}\right]}{a-b x_{1}^{*}} \times e^{(a-c) \tau}<1
$$

hold, then the periodic solution of system (2.1) is orbitally asymptotically stable.

Proof From condition (4.1) and Theorem 4.1, we know that there exists a $\tau$-periodic solution of system (2.1). Here

$$
\tau=\frac{1}{a} \ln \frac{(1-d) b x_{1}^{*}-a}{(1-d)\left(b x_{1}^{*}-a\right)} .
$$

System (2.1) can be rewritten as

$$
\left\{\begin{array}{l}
\frac{d x_{1}}{d t}=x_{1}\left(a-b x_{1}\right), \\
\frac{d x_{2}}{d t}=-c x_{2}, \\
\triangle x_{1}=-d x_{1}, \\
\triangle x_{2}=d x_{1},
\end{array}\right\} x_{1}-x_{1}^{*} \neq 0,
$$

We can easily derive

$$
\begin{aligned}
& P\left(x_{1}, x_{2}\right)=x_{1}\left(a-b x_{1}\right), \quad Q\left(x_{1}, x_{2}\right)=-c x_{2}, \\
& \phi\left(x_{1}, x_{2}\right)=x_{1}-x_{1}^{*}, \\
& \alpha\left(x_{1}, x_{2}\right)=-d x_{1}, \quad \beta\left(x_{1}, x_{2}\right)=d x_{1} .
\end{aligned}
$$

Then we calculate

$$
\begin{aligned}
\frac{\partial P}{\partial x_{1}} & =a-2 b x_{1}, \quad \frac{\partial Q}{\partial x_{2}}=-c, \quad \frac{\partial \alpha}{\partial x_{1}}=-d, \quad \frac{\partial \alpha}{\partial x_{2}}=0, \\
\frac{\partial \beta}{\partial x_{1}} & =d, \quad \frac{\partial \beta}{\partial x_{2}}=0, \quad \frac{\partial \phi}{\partial x_{1}}=1, \quad \frac{\partial \phi}{\partial x_{2}}=0, \\
\triangle_{1} & =\frac{P_{+}\left(\frac{\partial \beta}{\partial x_{2}} \frac{\partial \phi}{\partial x_{1}}-\frac{\partial \beta}{\partial x_{1}} \frac{\partial \phi}{\partial x_{2}}+\frac{\partial \phi}{\partial x_{1}}\right)+Q_{+}\left(\frac{\partial \alpha}{\partial x_{1}} \frac{\partial \phi}{\partial x_{2}}-\frac{\partial \alpha}{\partial x_{2}} \frac{\partial \phi}{\partial x_{1}}+\frac{\partial \phi}{\partial x_{2}}\right)}{P \frac{\partial \phi}{\partial x_{2}}+Q \frac{\partial \phi}{\partial x_{2}}} \\
& =\frac{P_{+}}{P}=\frac{P\left(\zeta\left(\tau_{k}^{+}\right), \eta\left(\tau_{k}^{+}\right)\right)}{P\left(\zeta\left(\tau_{k}\right), \eta\left(\tau_{k}\right)\right)} \\
= & \frac{(1-d) x_{1}^{*}\left[a-b(1-d) x_{1}^{*}\right]}{x_{1}^{*}\left[a-b x_{1}^{*}\right]} \\
= & \frac{(1-d)\left[a-b(1-d) x_{1}^{*}\right]}{a-b x_{1}^{*}} .
\end{aligned}
$$


From (3.4), it is also calculated

$$
\begin{aligned}
\mu_{2} & =\triangle_{1} e^{\int_{0}^{\tau_{0}}\left(\frac{\partial P}{\partial x_{1}}(\zeta(t), \eta(t))+\frac{\partial Q}{\partial x_{2}}(\zeta(t), \eta(t))\right) d t}=\frac{(1-d)\left[a-b(1-d) x_{1}^{*}\right]}{a-b x_{1}^{*}} \times e^{\int_{0}^{\tau}\left(a-2 b x_{1}(t)-c\right) d t} \\
& \leq \frac{(1-d)\left[a-b(1-d) x_{1}^{*}\right]}{a-b x_{1}^{*}} \times e^{(a-c) \tau} .
\end{aligned}
$$

In view of (4.7), it follows from Lemma 3.4 that the $\tau$-periodic solution of system (2.1) is orbitally asymptotically stable. This completes the proof.

\section{Discussion}

In this work, we investigate a single population model with state-dependent impulsively unilateral diffusion between two patches. From Theorem 4.1 and Theorem 4.3, we obtain the sufficient conditions of the existence of a periodic solution of system (2.1), and the sufficient conditions for orbitally asymptotic stability of a periodic solution of system (2.1) are also obtained. It is assumed that $x_{1}(0)=0.5, x_{2}(0)=0.3, x_{1}^{*}=1, a=3, b=1, c=1$, $d=0$, that is to say, there is no diffusion between these two patches; the time-series of $x_{1}(t)$ and $x_{2}(t)$ and the phase portrait of system (2.1) can be seen in Figure 1. We assume

(a)

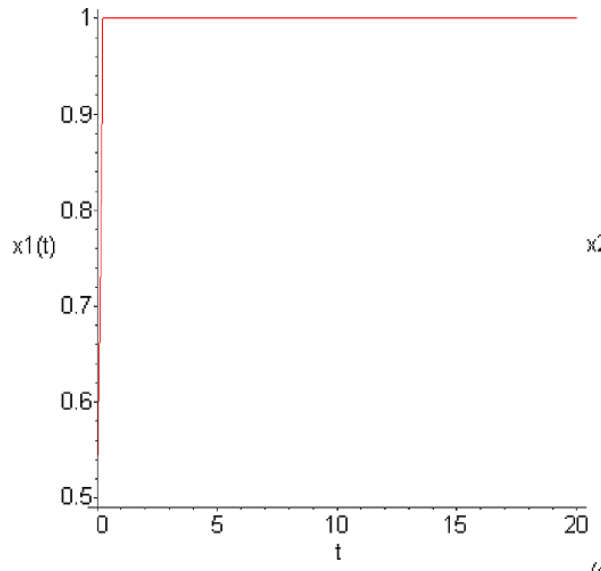

(c)

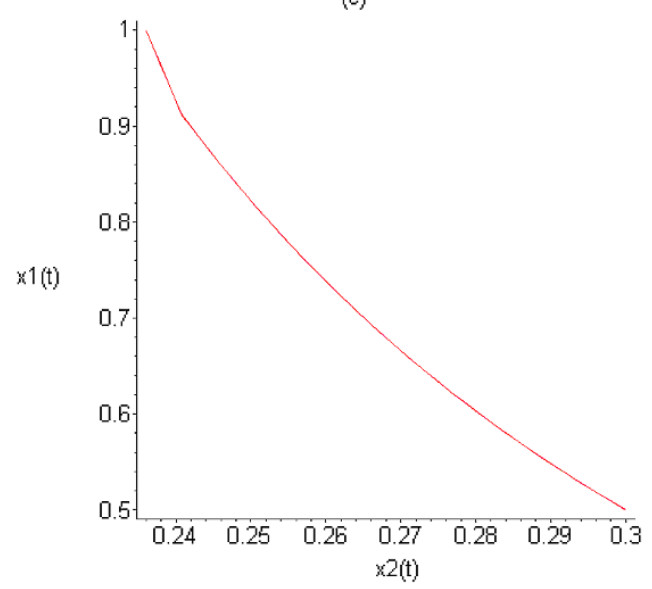

(b)

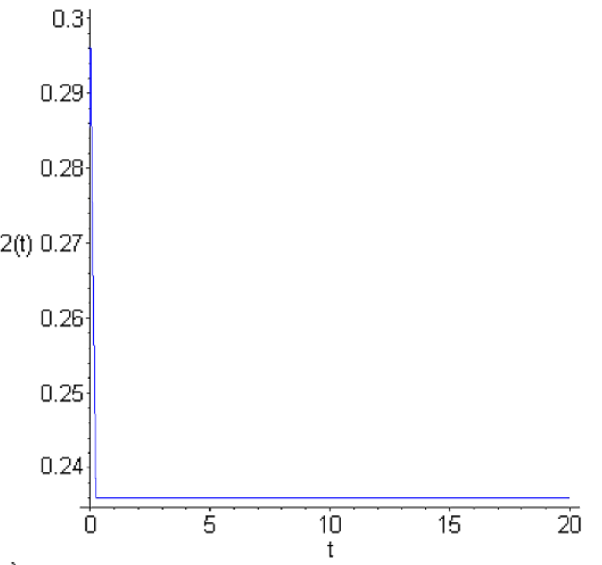

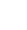


(a)

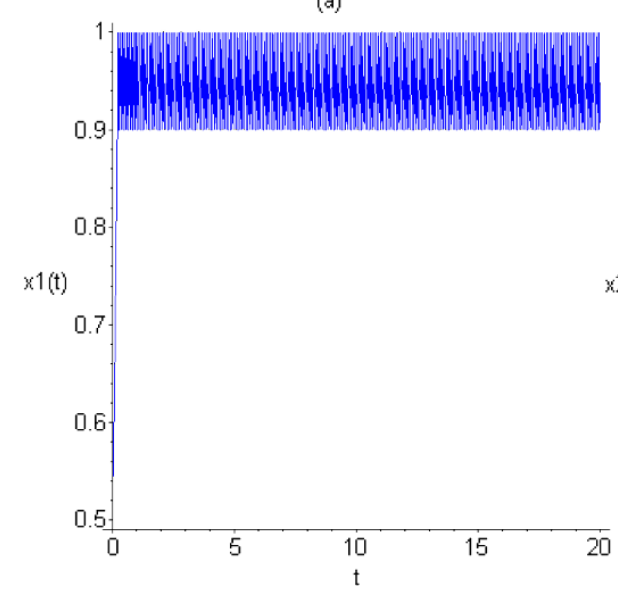

(b)

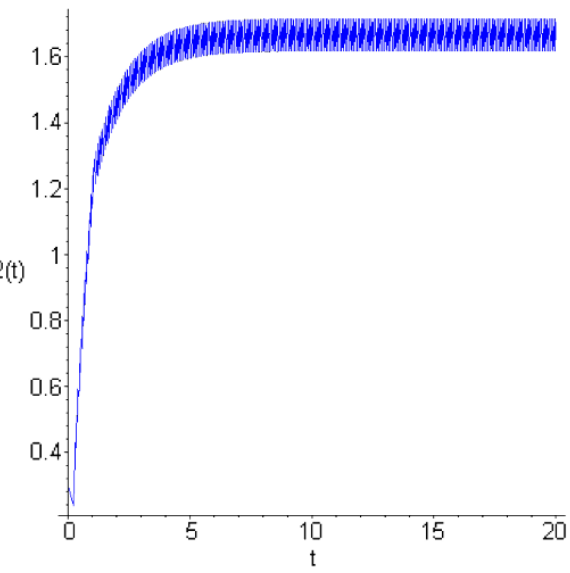

(c)

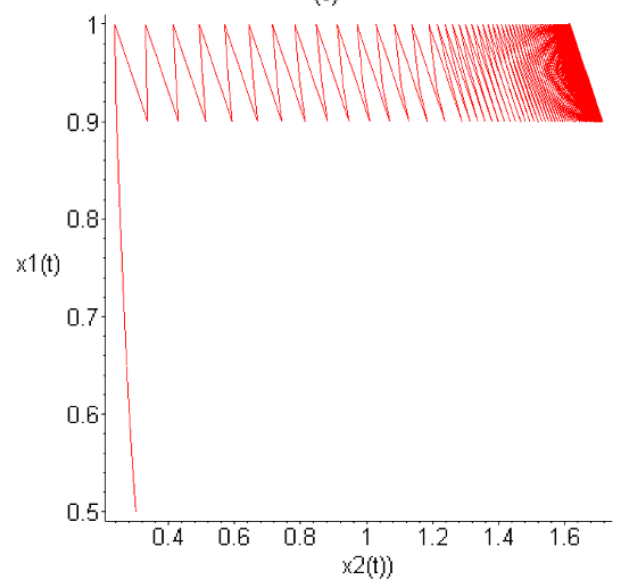

Figure 2 There is a small diffusion rate in system (2.1) with $x_{1}(0)=0.5, x_{2}(0)=0.3, x_{1}^{*}=1, a=3, b=1$, $c=1, d=0.1$, (a) time-series of $x_{1}(t)$; (b) time-series of $x_{2}(t)$; (c) the phase portrait of system (2.1).

that $x_{1}(0)=0.5, x_{2}(0)=0.3, x_{1}^{*}=1, a=3, b=1, c=1, d=0.1$, that is to say, there is a small diffusion rate between these two patches; the time-series of $x_{1}(t)$ and $x_{2}(t)$ and the phase portrait of system (2.1) can be seen in Figure 2. We also assume that $x_{1}(0)=0.5, x_{2}(0)=0.3$, $x_{1}^{*}=1, a=3, b=1, c=1, d=0.4$, that is to say, there is a relatively great diffusion rate between these two patches; the time-series of $x_{1}(t)$ and $x_{2}(t)$ and the phase portrait of system (2.1) can be seen in Figure 3. From the results of the numerical analysis, we can know that the diffusion plays an important role in the permanence of system (2.1), that is to say, when there is no diffusion between two patches, the population in Patch 2 will go extinct; when there is a relatively small diffusion rate, the population in Patch 2 will be permanent. Furthermore, for $d=0.1$ and $d=0.4$ with $x_{1}(0)=0.5, x_{2}(0)=0.3, a=3, b=1$, $c=1$, we know that these parameters satisfy the conditions of Theorem 4.3. The pictures in Figure 1 and Figure 2 show that there is existence and orbitally asymptotic stability of periodic solution of system (2.1). The numerical simulations verify our results. 
(a)

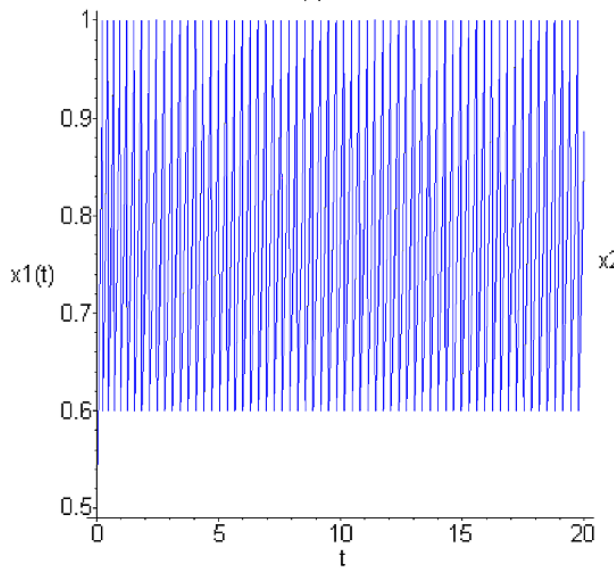

(b)

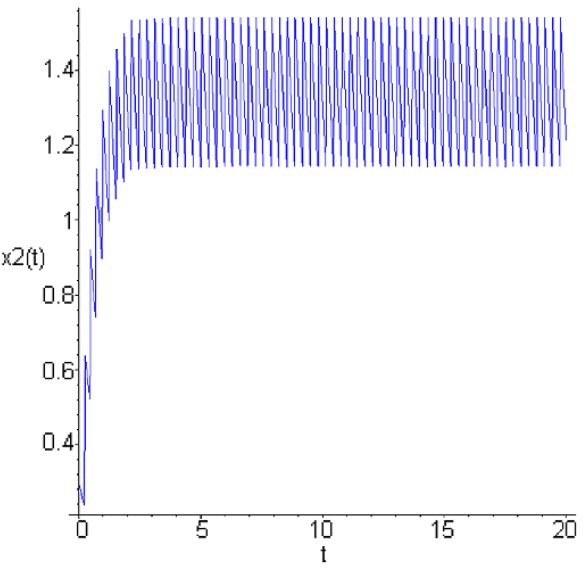

(c)

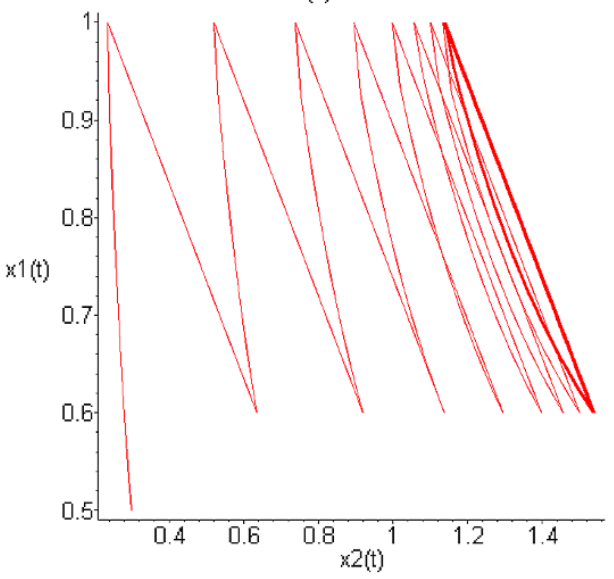

Figure 3 There is a small diffusion rate in system (2.1) with $x_{1}(0)=0.5, x_{2}(0)=0.3, x_{1}^{*}=1, a=3, b=1$, $c=1, d=0.4$, (a) time-series of $x_{1}(t)$; (b) time-series of $x_{2}(t)$; (c) the phase portrait of system (2.1).

\section{Competing interests}

The authors declare that they have no competing interests.

\section{Authors' contributions}

JJ carried out the main part of this article, LC corrected the manuscript, SC brought forward some suggestion on this article. All authors have read and approved the final manuscript.

\section{Author details}

${ }^{1}$ School of Mathematics and Statistics, Guizhou College of Finance and Economics, Guiyang, 550004, P.R. China. ${ }^{2}$ Institute of Mathematics, Academy of Mathematics and System Sciences, Beijing, 100080, P.R. China.

\section{Acknowledgements}

Research supported by National Natural Science Foundation of China (10961008), the Development Project of Nature Science Research of Guizhou Province Department (No. 2010027) and the Science Technology Foundation of Guizhou (No. 2010J2130).

Received: 17 April 2012 Accepted: 20 August 2012 Published: 10 September 2012

\section{References}

1. Levin, SA: Dispersion and population interactions. Am. Nat. 108, 207-228 (1974)

2. Cosner, C, Lou, Y: Does movement toward better environments always benefit a population. J. Math. Anal. Appl., 227, 489-503 (2003)

3. Lou, Y: Some challenging mathematical problems in evolution of dispersal and population dynamics. In: Friedman, A (ed.) Tutorials in Mathematical Biosciences IV: Evolution and Ecology. Springer, Berlin (2008)

4. Cui, JA: The effect of dispersal on population growth with stage structure. Comput. Math. Appl. 39, 91-102 (2000)

5. Takeuchi, Y: Diffusion-mediated persistence in three-species competition models with heteroclinic cycles. Math. Biosci. 106, 111-128 (1991) 
6. Abrams, PA, Cressman, R, Krivan, V: The role of behavioral dynamics in determining the patch distributions of interacting species. Am. Nat. 169, 505-518 (2007)

7. Kuang, Y, So, JWH: Analysis of a delayed two-stage population model with space limited recruitment. SIAM J. Appl. Math. 55, 1675-1696 (1995)

8. Lakshmikantham, V, Bainov, DD, Simeonov, P: Theory of Impulsive Differential Equations. World Scientific, Singapore (1989)

9. Bainov, D, Simeonov, P: Impulsive Differential Equations: Periodic Solutions and Applications. Pitman Monographs and Surveys in Pure and Applied Mathematics, vol. 66 (1993)

10. Jiao, JJ, Pang, GP, Chen, LS, Luo, GL: A delayed stage-structured predator-prey model with impulsive stocking on prey and continuous harvesting on predator. Appl. Math. Comput. 195(1), 316-325 (2008)

11. Tang, SY, Chen, LS: Multiple attractors in stage-structured population models with birth pulses. Bull. Math. Biol. 65 479-495 (2003)

12. Jiao, JJ, Cai, SH, Chen, LS: Analysis of a stage-structured predator-prey system with birth pulse and impulsive harvesting at different moments. Nonlinear Anal., Real World Appl. 12, 2232-2244 (2011)

13. Jiao, JJ, Chen, LS, Long, W: Pulse fishing policy for a stage-structured model with state-dependent harvesting. J. Biol. Syst. 15(3), 409-416 (2007)

14. Jiao, JJ, Chen, LS, Cai, SH, Wang, LM: Dynamics of a stage-structured predator-prey model with prey impulsively diffusing between two patches. Nonlinear Anal., Real World Appl. 41, 2748-2756 (2010)

15. Paneyya, JC: A mathematical model of periodically pulse chemotherapy: tumor recurrence and metastasis in a competition environment. Bull. Math. Biol. 58, 425-447 (1996)

doi:10.1186/1687-1847-2012-155

Cite this article as: Jiao et al.: Dynamical analysis on a single population model with state-dependent impulsively unilateral diffusion between two patches. Advances in Difference Equations 2012 2012:155.

\section{Submit your manuscript to a SpringerOpen ${ }^{\circ}$ journal and benefit from:}

- Convenient online submission

Rigorous peer review

- Immediate publication on acceptance

- Open access: articles freely available online

- High visibility within the field

- Retaining the copyright to your article 